\title{
Property C for Ordinary Differential Equations and Applications to Inverse Scattering
}

\author{
A. G. Ramm \\ Dedicated to Prof. L. von Wolfersdorf on the occasion of his retirement
}

\begin{abstract}
Property C, that is, completeness of the set of products of some solutions to SturmLiouville equations is proved. Several uniqueness theorems for various inverse scattering problems are obtained in a very simple way with the help of property $C$. Two classical uniqueness results for inverse scattering problem on the half-axis and for the inverse spectral problem are proved in a very short new way. Earlier the author introduced property $\mathrm{C}$ for partial differential equations and used it extensively for proving uniqueness theorems for many inverse problems.
\end{abstract}

Keywords: Property $C$, inverse scattering, non-destructive testing, remote sensing, SturmLiouville equation

AMS subject classiflcation: $35 \mathrm{R} 30$

\section{Introduction}

Property $\mathrm{C}$ was introduced in [5], applied to inverse scattering for partial differential equations in [6] and the theory is developed systematically in [7], where its many applications to inverse problems are presented. In [3] some results on completeness of the set of products of solutions to pairs of Sturm-Liouville equations are obtained and applied to some problems for ordinary differential equations. In [13] a new method is given for derivation of the completeness results for products of solutions to ordinary differential equations and examples of incompleteness of the set of products of solutions to ordinary differential equations are given. These results were related to some inverse scattering problems of geophysics [9].

In this paper we use the idea from [13] but simplify and generalize it and obtain new results on completeness of the set of products of solutions to ordinary differential equations. We apply these results to several inverse scattering problems. In particular, we give a new very short proof of the uniqueness of recovery of the potential from $I$ function $[7,8]$. This uniqueness result can be obtained from the uniqueness results for the inverse problem with spectral function as data (see $[3,8]$ ) but our proof is much shorter. We derive a uniqueness theorem for finding a compactly supported potential from the boundary data known at all energies and several other uniqueness theorems for various inverse problems. The statements of these inverse problems and the proofs

A. G. Ramm: Kansas State Univ., Dept. Math., Manhattan, KS 66506-2602, USA e-mail: ramm@math.ksu.edu. The author thanks DAAD for support. 
of the uniqueness theorems for them are given in Section 3. In Section 2 property $C$ for some pairs of ordinary differential equations is established. In Section 4 a new short proof is given for the classical inverse scattering problem on the half-axis and in Section 5 a uniqueness theorem is proved for the inverse problem with mixed data: the potential is known on a part of the interval and part of the spectrum of the associated Sturm-Liouville operator is given.

\section{Property $\mathrm{C}$ for ordinary differential equations}

Let

$$
\ell u:=u^{\prime \prime}+k^{2} u-q(x) u=0 \quad(-\infty<x<\infty)
$$

where $k=$ const, $q$ is a real-valued function, $\int_{-\infty}^{\infty}(1+|x|)|q(x)| d x<\infty$. The scattering problem consists in finding the solution to (2.1) with the asymptotics

$$
u= \begin{cases}\exp (i k x)+r(k) \exp (-i k x)+o(1) & \text { for } x \rightarrow-\infty \\ t(k) \exp (i k x) & \text { for } x \rightarrow+\infty\end{cases}
$$

The coefficients $r(k)$ and $t(k)$ are called the reflection and transmission coefficients. The Jost solutions of (2.1) are the solutions with the asymptotics

$$
f_{ \pm}(x, k)=\exp ( \pm i k x)+o(1) \quad(x \rightarrow+\infty)
$$

and

$$
g_{ \pm}(x, k)=\exp ( \pm i k x)+o(1) \quad(x \rightarrow-\infty) .
$$

Denote by $\ell_{j}(j=1,2)$ operators $(2.1)$ with $q=q_{j}(x)$, let $\mathbb{R}_{+}=(0, \infty), \mathbb{R}_{-}=(-\infty, 0)$, $L_{1,1}(\Delta):=\left\{q: \int_{\Delta}(1+|x|)|q(x)| d x<\infty\right\}$.

Definition 2.1. We say that the pair $\left\{\ell_{1}, \ell_{2}\right\}$ has property $C_{ \pm}$if the sets

$$
\left\{f_{1}(x, k) f_{2}(x, k)\right\}_{0<k<\infty} \quad \text { and } \quad\left\{g_{1}(x, k) g_{2}(x, k)\right\}_{0<k<\infty}
$$

are complete in $L_{1,1}\left(\mathbb{R}_{ \pm}\right)$, respectively.

Here $f_{j}$ and $g_{j}$ are $f_{j+}$ and $g_{j+}$, respectively, where $f_{+}$and $g_{+}$are defined by formulas (2.3) and (2.4). We assume throughout that $q \in L_{1,1}(-\infty, \infty)$.

Theorem 2.1. The pair $\left\{\ell_{1}, \ell_{2}\right\}$ has property $C_{ \pm}$.

Proof. We prove property $C_{+}$. Proof of $C_{-}$is similar. The idea of the proof is to reduce the problem of completeness to the problem of injectivity of a certain Volterra operator. It is well known (see $[4,7])$ that

$$
f_{j}(x, k)=\exp (i k x)+\int_{x}^{\infty} A_{j}(x, t) \exp (i k t) d t
$$

where $f_{j}$ is the Jost solution for $\ell_{j}$, and the kernels $A_{j}(x, t)$ are continuous functions, such that

$$
\sup _{x \geq 0} \int_{x}^{\infty}|A(x, t)| d t<\infty \text { and } \quad|A(x, y)| \leq c \int_{\frac{x+y}{2}}^{\infty}|q| d t:=c \sigma\left(\frac{x+y}{2}\right)
$$


and their derivatives $A_{x}$ and $A_{y}$ are absolutely integrable with respect to $y$ over the interval $(x, \infty)$ for any $x \geq 0$. We have

$$
\begin{aligned}
f_{1} f_{2}= & \exp (2 i k x)+\int_{x}^{\infty} B(x, t) \exp [i k(t+x)] d t \\
& +\int_{x}^{\infty} \int_{x}^{\infty} A_{1}(x, t) A_{2}(x, \tau) \exp [i k(t+\tau)] d t d \tau
\end{aligned}
$$

where

$$
B(x, t):=A_{1}(x, t)+A_{2}(x, t),
$$

and, setting $t+\tau=s, t-\tau=\sigma$, one gets

$$
\int_{x}^{\infty} \int_{x}^{\infty} A_{1}(x, t) A_{2}(x, \tau) \exp [i k(t+\tau)] d t d \tau=\int_{2 x}^{\infty} T(x, s) \exp (i k s) d s,
$$

where

$$
T(x, s)=\frac{1}{2} \int_{-(s-2 x)}^{s-2 x} A_{1}\left(x, \frac{s+\sigma}{2}\right) A_{2}\left(x, \frac{s-\sigma}{2}\right) d \sigma .
$$

Suppose $h \in L_{1,1}\left(\mathbb{R}_{+}\right)$and

$$
0=\int_{0}^{\infty} h(x) f_{1}(x, k) f_{2}(x, k) d x \quad \forall k>0 .
$$

Then, using (2.7) and (2.10) one gets

$$
\begin{aligned}
0= & \int_{0}^{\infty} d x e^{2 i k x} h(x)+\int_{0}^{\infty} d s e^{i k s} \int_{0}^{\frac{1}{2}} B(x, s-x) h(x) d x \\
& +\int_{0}^{\infty} d s e^{i k s} \int_{0}^{\frac{1}{2}} T(x, s) h(x) d x
\end{aligned}
$$

or

$$
0=\int_{0}^{\infty} d s e^{2 i k s}\left[h(s)+2 \int_{0}^{s} B(x, 2 s-x) h(x) d x+2 \int_{0}^{s} T(x, 2 s) h(x) d x\right]
$$

for all $k>0$. The right-hand side of (2.12) is analytic with respect to $k$ in the upper half-plane $\operatorname{Im} k>0$ and vanishes for $k>0$. Thus, it has to vanish for $k<0$ as well. Therefore (2.12) implies

$$
h(s)+2 \int_{0}^{s} B(x, 2 s-x) h(x) d x+2 \int_{0}^{s} T(x, 2 s) h(x) d x=0 \quad \forall s>0 .
$$

Since $B(x, 2 s)$ and $T(x, 2 s)$ are bounded continuous functions, it follows from the Volterra equation (2.13) that $h(s)=0$. Theorem 2.1 is proved

Let us prove another theorem of the property $C$ type. Let $\phi_{j}(x, k)$ be the solutions to equation (2.1) with $\ell=\ell$; which satisfies the conditions $\phi(0, k)=0, \phi^{\prime}(0, k)=k$. Similar considerations are valid for the functions $\psi(x, k)$, which solve equation $(2.1)$ and satisfy the conditions $\psi^{\prime}(0, k)=0, \psi(0, k)=1$. Fix an arbitrary number $b>0$. 
Theorem 2.2. The sets of products

$$
\left\{\phi_{1}(x, k) \phi_{2}(x, k)\right\}_{k>0} \quad \text { and } \quad\left\{\psi_{1}(x, k) \psi_{2}(x, k)\right\}_{k>0}
$$

are complete in $L^{2}(0, b)$.

Proof. The idea of the proof is similar to that of Theorem 2.1. We have

$$
\phi_{j}(x, k)=\sin (k x)+\int_{0}^{x} K_{j}(x, y) \sin (k y) d y \quad(j=1,2),
$$

where the kernels $K_{j}$ define the operators (see $[3,4]$ and [7: p. 250]), which transform the solution to equation (2.1) with $q(x)=0$, satisfying the above initial conditions at $x=0$, into the solution to equation (2.1) with $q=q_{j}$, satisfying the same initial conditions. Thus

$$
\begin{aligned}
\phi_{1} \phi_{2}= & \sin ^{2}(k x)+\int_{0}^{x} K(x, y) \sin (k x) \sin (k y) d y \\
& +\frac{1}{2} \int_{0}^{x} \int_{0}^{x} K_{1}(x, y) K_{2}(x, s)\{\cos [k(y-s)]-\cos [k(y+s)]\} d y d s,
\end{aligned}
$$

where $K:=K_{1}+K_{2}$. Assume that $0=\int_{0}^{b} d x f(x) \phi_{1} \phi_{2}$ for all $k>0$. Then

$$
\begin{aligned}
0= & \int_{0}^{b} d x f(x)-\int_{0}^{b} d x f(x) \cos (2 k x) \\
& +\int_{0}^{b} d s \cos (k s) \int_{s}^{b} d x f(x) K(x, x-s) \\
& -\int_{0}^{2 b} d s \cos (k s) \int_{\frac{3}{2}}^{\min (b, s)} d x f(x) K(x, s-x)+I,
\end{aligned}
$$

where

$$
I:=\int_{0}^{b} d x f(x) \int_{0}^{x} \int_{0}^{x} K_{1}(x, y) K_{2}(x, s)\{\cos [k(y-s)]-\cos [k(y+s)]\} d y d s .
$$

Let $y-s:=t, y+s:=v$. Then

$$
\int_{0}^{x} \int_{0}^{x} K_{1} K_{2} \cos [k(y-s)] d y d s=\int_{0}^{x} d s \cos (k s) B_{1}(x, s),
$$

where

$$
\begin{aligned}
& B_{1}(x, s):=\frac{1}{2} \int_{|s|}^{2 x-|s|}\left[K_{1}\left(x, \frac{s+v}{2}\right) K_{2}\left(x, \frac{v-s}{2}\right)+K_{1}\left(x, \frac{v-s}{2}\right) K_{2}\left(x, \frac{v+s}{2}\right)\right] d v \\
& \int_{0}^{x} \int_{0}^{x} K_{1} K_{2} \cos [k(y+s)] d y d s=\int_{0}^{2 x} B_{2}(x, s) \cos (k s) d s \\
& B_{2}(x, s):=\frac{1}{2} \int_{-\omega(s)}^{\omega(s)} K_{1}\left(x, \frac{t+s}{2}\right) K_{2}\left(x, \frac{s-t}{2}\right) d t,
\end{aligned}
$$


and $\omega=s$ for $0 \leq s \leq x, \omega=2 x-s$ for $x \leq s \leq 2 x$. Therefore

$$
I=\int_{0}^{b} d s \cos (k s) \int_{s}^{b} d x f(x) B_{1}(x, s)-\int_{0}^{2 b} d s \cos (k s) \int_{\frac{1}{2}}^{b} d x f(x) B_{2}(x, s) .
$$

From (2.15) and (2.16), taking $k \rightarrow \infty$, one gets $\int_{0}^{b} f(x) d x=0$, and (using completeness of the system $\{\cos (k s)\}_{0<k<\infty}$ in $\left.L^{2}(0, b)\right)$ the following equation:

$$
\begin{aligned}
0= & -\frac{f\left(\frac{s}{2}\right)}{2}+\int_{s}^{b} K(x, x-s) f(x) d x-\int_{\frac{1}{2}}^{\min (b, s)} d x f(x) K(x, s-x) \\
& +\int_{s}^{b} d x f(x) B_{1}(x, s)-\int_{\frac{1}{2}}^{b} d x f(x) B_{2}(x, s) .
\end{aligned}
$$

The kernels $K, B_{1}$ and $B_{2}$ are bounded and continuous functions. Therefore, if $b<\infty$ and $f(x)=0$ for $x>b$, equation (2.17) implies

$$
|f(y)| \leq c \int_{2 y}^{b}|f(x)| d x+c \int_{y}^{b}|f(x)| d x
$$

where $c>0$ is a constant which bounds the kernels $2 K, 2 B_{1}$ and $2 B_{2}$ from above and $2 y=s$. From the above inequality one gets

$$
\max _{b-\epsilon \leq y \leq b}|f(y)| \leq c \epsilon \max _{b-\epsilon \leq y \leq b}|f(y)|
$$

where $\epsilon \quad(0<\epsilon<b)$ is sufficiently small so that $c \epsilon<1$ and $b-\epsilon<2 b-2 \epsilon$. Then inequality (2.18) implies $f(x)=0$ if $b-\epsilon<x<b$. Repeating this argument, one proves, in finitely many steps, that $f(x)=0$ if $0<x<b$. Theorem 2.2 is proved

Remark 2.1. The proof of Theorem 2.2 is not valid if $b=\infty$.

\section{Applications}

3.1. Consider the following inverse scattering problem which often arises in geophysics. Assume $q(x)=0$ for $x<0$, suppose that the scattering problem (2.2) is considered, and the scattering data is the impedance function, the $I(k)$ function, defined as

$$
I(k)=\frac{u^{\prime}(0, k)}{u(0, k)} \quad(k>0)
$$

Problem (IP1). The inverse scattering problem is: given $I(k)$ for all $k>0$, recover $q \in L_{1,1}\left(\mathbb{R}_{+}\right)$.

If $q(x)=0$ for $x<0$, then (2.2) implies

$$
u^{\prime}(0, k)=i k(1-r(k)) \quad \text { and } \quad u(0, k)=1+r(k)
$$


so

$$
I(k)=i k \frac{1-r(k)}{1+r(k)} .
$$

Therefore $I(k)$ and $r(k)$ are in one-to-one correspondence, and problem (IP1) can be reduced to the inverse scattering on the full line which is developed in [4]. However, the inverse scattering theory on the full line uses as the scattering data not only the reflection coefficient, but also the bound states and the norming constants. Therefore this theory is not applicable since we do not know the bound states and the norming constants.

From Theorem 3.1, which we prove below, it follows that if one knows a priori that $q(x)=0$ for $x<0$, then the knowledge of the reflection coefficient alone determines uniqucly the potential $q \in L_{1,1}$, and therefore, determines the bound states and the norming constants (see also $[8,10,11]$ ).

Another approach to problem (IP1) which gives an inversion procedure and necessary and sufficient conditions for a given function $I(k)$ to be an $I$-function corresponding to a potential $q$ from some functional class (for instance, $m$ times differentiable $q$ ) is given in [8].

Here our goal is to give a very short proof of the uniqueness of the solution to problem (IP1). This proof is patterned after the proofs of several uniqueness theorems in [7], and we want to emphasize the method of the proof.

Theorem 3.1. Problem (IP1) has at most one solution.

Proof. Suppose there are two potentials $q_{j}(j=1,2)$ with the same $I(k)$ and $u_{j}(x, k)$ are the corresponding scattering solutions,

$$
\ell_{j} u_{j}=0
$$

where $\ell_{j}$ is the operator (2.1) with $q=q_{j}(j=1,2)$. Note that (2.2) implies

$$
u_{j}=t_{j}(k) f_{j}(x, k)
$$

so

$$
I_{j}(k)=\frac{f_{j}^{\prime}(0, k)}{f_{j}(0, k)} \quad(j=1,2),
$$

where $f_{j}(x, k)=f_{j+}(x, k)$ are the Jost solutions, defined by formula (2.5):

$$
\ell_{j} f_{j}=0 \quad(j=1,2)
$$

Subtract from (3.7) with $j=1$ equation (3.7) with $j=2$ and get

$$
\ell_{1} w=p f_{2}, \quad w:=f_{1}-f_{2}, p:=q_{1}-q_{2} .
$$

Let us assume now that

$$
I_{1}(k)=I_{2}(k) \quad(k>0)
$$


and derive from (3.8) and (3.9) that

$$
p(x)=0,
$$

which is equivalent to the desired conclusion:

$$
q_{1}(x)=q_{2}(x) \text {. }
$$

This derivation is simple: multiply $(3.8)$ by $f_{2}(x, k)$ and integrate over $(0, \infty)$ to get

$$
\int_{0}^{\infty} p f_{1} f_{2} d x=\int_{0}^{\infty} \ell_{1} w f_{1} d x=\int_{0}^{\infty} w \ell_{1} f_{1} d x+\left.\left(w^{\prime} f_{1}-w f_{1}^{\prime}\right)\right|_{0} ^{\infty}=0
$$

and the last equation will be explained below. From Theorem 2.1 and the orthogonality condition, which follows from equation (3.12):

$$
\int_{0}^{\infty} p(x) f_{1}(x, k) f_{2}(x, k) d x=0 \quad(k>0)
$$

the desired conclusion (3.10) follows immediately.

To finish this very short proof, let us explain that the integral on the right-hand side of (3.12) vanishes since $\ell_{1} f_{1}=0$, and the boundary terms in (3.12) vanish because

1) $w=w^{\prime}=0$ at infinity since $f_{1}$ and $f_{2}$ have the same asymptotics as $x \rightarrow+\infty$ and

2) $G:=w^{\prime}(0, k) f_{1}(0, k)-w(0, k) f_{1}^{\prime}(0, k)=0$ because of $(3.9)$.

Indeed,

$$
G=f_{1}^{\prime} f_{1}-f_{2}^{\prime} f_{1}-f_{1} f_{1}^{\prime}+f_{2} f_{1}^{\prime}=f_{1}^{\prime} f_{2}-f_{2}^{\prime} f_{1}=f_{1} f_{2}\left(\frac{f_{1}^{\prime}}{f_{1}}-\frac{f_{2}^{\prime}}{f_{2}}\right)=0 .
$$

Theorem 3.1 is proved

3.2. As the second application of Theorem 2.1 and the above method of proof, consider the following inverse scattering problem. Let

$$
\ell g=-\delta(x-y), \quad \frac{\partial g}{\partial|x|}-i k g \rightarrow 0 \text { as }|x| \rightarrow \infty
$$

where $\ell$ is defined in (2.1), $\delta(x)$ is the delta-function, and $y<0$. Assume that $y$ is fixed and

$$
q \in L_{1}[0, a], \quad q(x)=0 \text { if } x \notin[0, a] \quad(0<a<\infty) .
$$

As the scattering data take the values

$$
v_{0}(k):=g(0, k) \quad \text { and } \quad v_{1}(k):=g(a, k) \quad(k>0) .
$$

Problem (IP2). Do the data (3.17) determine $q(x)$ uniquely?

One can consider different data, for example, $I_{0}(k):=\frac{g^{\prime}(0, k)}{g(0, k)}$ and $I_{1}(k):=\frac{g^{\prime}(a, k)}{g(a, k)}$; or any boundary conditions which imply that the boundary term, analogous to the one in formula (3.12), vanishes. 
Theorem 3.2. The data (3.17) determine $q(x)$ uniquely.

Proof. Suppose $q_{j}(j=1,2)$ generate the same data. Then, as in the proof of Theorem 3.1,

$$
\ell_{1} w=p g_{2}, \quad w:=g_{1}-g_{2}, p:=q_{1}-q_{2} .
$$

Since $g_{1}$ and $g_{2}$ satisfy the radiation condition at $\pm \infty$ and $w(0, k)=w(a, k)=0$, one concludes that

$$
w(x, k)=0 \quad \text { if } \quad x \notin[0, a] .
$$

If $x \geq 0$, one has

$$
g_{2}(x, k)=c(k) f_{2}(x, k) \quad(c(k) \neq 0, x \geq 0)
$$

because $\ell_{2} g_{2}=0$ for $x \geq 0$ and $g_{2}$ satisfies the radiation condition (3.15). Multiply (3.18) by $f_{1}(x, k)$, integrate over $(-\infty, \infty)$ and then integrate by parts using (3.19) and (3.20) to get the orthogonality condition (3.13). Apply Theorem 2.1 and conclude that $p(x)=0$. Theorem 3.2 is proved

3.3. Consider the scattering problem on the full line assuming that $q \in L^{1}(0, a)$, $q(x)=0$ if $x \notin[0, a], q$ is real-valued. The scattering solution $u(x, k)$ is defined by equation (2.2). The non-standard scattering data we give are

$$
\{u(0, k), u(a, k)\}_{k>0} \text {. }
$$

These data are practically important. One can think about a layer of thickness $a$ and the field measured at the entrance into the layer and at the exit from the layer. From the measured field at all frequencies one wants to recover the function $q$ describing the layer.

Theorem 3.3. Let $u(x, k)$ be the scattering solution defined by (2.2). Then data (3.21) determine $q(x)$ uniquely.

Proof. As in the proof of Theorem 3.2 one gets an equation similar to (3.18):

$$
\ell_{1} w=p u_{2}, \quad w:=u_{1}-u_{2}, p:=q_{1}-q_{2}
$$

and

$$
w(0, k)=w(a, k)=0 \quad(\forall k>0) .
$$

If $p(x)=0$ for $x>a$, then

$$
w^{\prime \prime}(x, k)+k^{2} w(x, k)=0 \quad(x>a)
$$

Therefore

$$
w=c_{1}(k) \exp (i k x)+c_{2}(k) \exp (-i k x) .
$$

Since $w$ is a difference of two functions each of which satisfies the second relation (2.2), it follows that $c_{2}(k)=0$ and $w=c_{1}(k) \exp (i k x)$. If $w(a, k)=0$, then $c_{1}(k)=0$. Therefore $w(x, k)=0$ for $x>a$. 
Similarly one proves, using the first condition (2.2), that $w(0, k)=0$ implies $w(x, k)=0$ for $x<0$. Therefore

$$
w(x, k)=0 \quad \text { if } x \notin[0, a] .
$$

From (2.2) it follows that

$$
u_{2}=t_{2}(k) f_{2}(x, k) \text {. }
$$

Multiply (3.22) by $f_{1}(x)$ and integrate over $[0, a]$ to get

$$
0=\int_{0}^{a} p(x) u_{2}(x, k) f_{1}(x, k) d x \quad(\forall k>0)
$$

From (3.26), (3.25) and Theorem 2.1 it follows that $p(x)=0$. Theorem 3.3 is proved

Remark 3.1. Note that Theorem 3.1 implies that the data $\{u(0, k)\}_{k>0}$ alone determine $q(x)$ which vanishes outside the interval $[0, a]$. Indeed, if $u(x, k)$ is the scattering solution (2.2), and $q=0$ for $x<0$, then $u=e^{i k x}+r(k) e^{-i k x}$ for $x<0$. Thus, $u(0, k)=1+r(k)$, so the data $\{u(0, k)\}_{k>0}$ determine $r(k)$ for all $k>0$, and Theorem 3.1 guarantees that $q(x)$ is uniquely determined by these data because $r(k)$ determines uniquely $I(k)$ if $q(x)=0$ for $x<0$. To see this, note that the solution to (2.2) equals to $t(k) f(x, k)$ for $x>0$, so $\frac{u^{\prime}(0, k)}{u(0, k)}=I(k)$, and $u=e^{i k x}+r(k) e^{-i k x}$ for $x<0$, so $I(k)=\frac{u^{\prime}(0, k)}{u(0, k)}=\frac{i k[1-r(k)]}{1+r(k)}$, which is formula (3.3). Therefore $r(k)$ determines uniquely $I(k)$ if $q(x)=0$ for $x<0$, and Theorem 3.1 is applicable.

On the other hand, it is not clear (and is not likely in general) that the data at $x=a$ alone determine $q(x)$ uniquely. Indeed, these data will determine the transmission coefficient $t(k)$, rather than the reflection coefficient, and the problem of the recovery of a compactly supported potential from the transmission coefficient alone was not studied in the literature to our knowledge.

3.4. Let $q$ be the same as in Theorem 3.3. In the same way as Theorem 3.3 one proves

Theorem 3.4. Let $u(x, k)$ be the scattering solution defined by formula (2.2). Then the data

determine $q(x)$ uniquely.

$$
\left\{u_{x}^{\prime}(0, k), u(a, k)\right\}_{\forall k>0}
$$

Remark 3.2. a) Other data containing linear combinations of $u(a, k)$ and $u^{\prime}(a, k)$ can be to use.

b) The data can be given not for all $k>0$ but for $k$ on an arbitrary small open subset of the positive semiaxis $(0, \infty)$. Indeed, if the potential is compactly supported, then $u(x, k)$ known on an open subset of the positive semiaxis $k>0$ determines uniquely the values of $u(x, k)$ for all $k>0$ by analyticity.

The uniqueness results will be valid in all the problems in which the data are the boundary values of functions, analytic in the half-plane $\operatorname{Im} k>0$ : such functions cannot vanish on an open subset of the real axis unless they vanish identically. The problems in Section 3 have this property.

3.5. As another example of many applications of property $\mathrm{C}$ for ordinary differential equations, we derive the following uniqueness theorem for inverse scattering by a potential $q$ on the whole line. 
Theorem 3.5. If $q \in L_{1,1}(-\infty, \infty)$, and $q(x)=0$ for $x<a$, where $a>-\infty$ is an arbitrary fixed number, then the data $\{r(k), t(k)\}_{k>0}$ determine $q$ uniquely.

Theorem 3.5 is weaker than the well-known uniqueness theorem for inverse scattering on the whole line, which does not require that $q(x)$ vanishes for $x<a$. However, our proof is very short and simple, and by this reason it is included here. The al1thor thinks that a modification of Theorem 2.1 will yield the uniqueness theorem, similar to Theorem 3.5, for any $q \in L_{1,1}(-\infty, \infty)$.

On the other hand, Remark 3.1 is applicable, and for potentials which vanish on the half-axis $x<0$ (one can take $a=0$ without loss of generality) the argument given in Remark 3.1 shows that the data $\{r(k)\}_{\forall k>0}$ determines $q$ uniquely, so the data in Theorem 3.5 for such potentials are overdetermined.

Proof of Theorem 3.5. As above, assuming that there are two potentials with the same data $\{r(k), t(k)\}_{k>0}$, one gets (3.22). Multiply (3.22) by $f_{1}(x, k)$ and integrate over $(-\infty, \infty)$ to get the orthogonality relation, similar to $(3.26)$ :

$$
\int_{-\infty}^{\infty} p(x) u_{2}(x, k) f_{1}(x, k) d x=0 \quad(\forall k>0) .
$$

The terms due to the integration by parts vanish, because $r_{1}=r_{2}$ and $t_{1}=t_{2}$ by the assumption. From (3.25), (3.28) and Theorem 2.1 it follows that $p=0$. Theorem 3.5 is proved

Remark 3.2. The reason we require $q(x)=0$ for $x<a$ in the above argument is that formula (2.5) is proved for $x>-\infty$.

In Theorem 3.5 the transmission coefficient $t(k)$ can be dropped from the data if $q(x)=0$ for $x<a$, and the conclusion of this theorem remains valid. This follows from the argument in Remark 3.1 .

3.6. In this subsection we give an application to an inverse problem for partial differential equations. Let $u=u(x, t)$,

$$
\begin{gathered}
u_{\ell t}=u_{x x}-q(x) u \quad(x>0, t>0) \\
u=u_{t}=0 \quad \text { at } t=0 \\
u(0, t)=\delta(t)
\end{gathered}
$$

where $\delta$ is the delta-function. The extra data are

$$
u(a, t)=f(t) \quad(\forall t>0)
$$

Assume that $q \in L_{1}\left(\mathbb{R}_{+}\right)$, and $q(x)=0$ for $x>a$ and for $x<0$. The inverse problem is Problem (IP3). Given $f$, find $q$. 
Theorem 3.6. The above inverse problem has at most one solution.

Proof. Taking Laplace transform of (3.29) - (3.32) one gets

$$
\begin{gathered}
v_{x x}-\lambda^{2} v-q(x) v=0 \quad(x>0, \lambda>0) \\
v(0, \lambda)=1 \\
v(a, \lambda)=F(\lambda)
\end{gathered}
$$

where

$$
v(x, \lambda):=\int_{0}^{\infty} u(x, t) \exp (-\lambda t) d t \quad \text { and } \quad F(\lambda):=\int_{0}^{\infty} f(t) \exp (-\lambda t) d t .
$$

The function $v(x, \lambda)$ is analytic in the half-plane $\operatorname{Re} \lambda>0$ and has limiting values on the imaginary axis $\operatorname{Re} \lambda=0$. Let $\lambda=i k, k>0$. Then equation (3.33) takes the form (2.1) and we denote the solution $v$ as a function of $k$ also by $v$. The solution $v(x, k)=\frac{f(x, k)}{f(k)}$, where $f(x, k)$ is the Jost solution to equation of (2.1). Indeed, the above. $v$ solves (2.1) and satisfies condition (3.34). Since $q(x)=0$ for $x>a$, one has $f(x, k)=e^{i k x}$ for $x>a$. Therefore condition (3.35) yields $f(k)$, namely $f(k)=\frac{e^{i a k}}{F(i k)}$. Therefore Theorem 3.6 follows from the result in [10] (see also [11])

One could give a proof of Theorem 3.6 based on property $\mathrm{C}$, but we want to demonstrate a different proof. The result in [10] says that a compactly supported real-valued integrable potential is uniquely determined by the Jost function $f(k)$ and there is no need to know a priori the bound states and norming constants: these are uniquely determined by $f(k)$ if the potential is compactly supported.

Recently the author solved the inverse problem of recovery of a compactly supported $q(x)$ from $\left\{f^{\prime}(0, k)\right\}_{\forall k>0}$.

3.7. Consider the problem

$$
\begin{gathered}
u_{t}=u_{x x}-q(x) u, \quad(0 \leq x \leq 1, t>0) \\
u_{x}(0, t)=0, \quad u_{x}(1, t)=b(t) \\
u(x, 0)=0 .
\end{gathered}
$$

The extra data are:

$$
u(1, t)=a(t) \quad(t>0) .
$$

Problem (IP4). The inverse problem is:

Assume that $b(t) \not \equiv 0$ is integrable and compactly supported. Given $a(t)$ and $b(t)$, find $q(x)$.

Theorem 3.7. The above problem (IP4) has at most one solution.

Proof. Let us transform problem (IP4) into an inverse problem for ordinary differential equations. To do this, take the Laplace transform

$$
v(x, \lambda):=\int_{0}^{\infty} \exp (-\lambda t) u(x, t) d t
$$


and get

$$
\begin{gathered}
v^{\prime \prime}-\lambda v-q(x) v=0 \quad(0 \leq x \leq 1) \\
v_{x}(0, \lambda)=0, \quad v_{x}(1, \lambda)=B(\lambda) \\
v(1, \lambda)=A(\lambda),
\end{gathered}
$$

where $A$ and $B$ are the Laplace transforms of $a$ and $b$, respectively. Assume that there are two potentials $q_{1}$ and $q_{2}$ with the same data (3.43), (3.44), $v_{1}:=v_{1}(x, \lambda)$ and $v_{2}:=v_{2}(x, \lambda)$ are the corresponding solutions to (3.42) - (3.44) and let $p:=q_{1}-q_{2}$, $w:=v_{1}-v_{2}$. From the first equation (3.43) it follows that $v=c(\lambda) \psi(x, \lambda)$, where $\psi(x, \lambda)$ solves (3.42) and satisfies the conditions $\psi(0, \lambda)=1, \psi_{x}(0, \lambda)=0$, and $c(\lambda)$ does not depend on $x$. Since $b(t)$ is compactly supported and $b(t) \not \equiv 0$, it follows that $B(\lambda)$ is an entire function of $\lambda$, therefore it may vanish at most at a discrete set of points. Note that this is valid even if $b(t)$ is a compactly supported distribution, for example the delta-function.

Problem (3.42) - (3.43) is uniquely solvable for all $\lambda$ except possibly at $\lambda$ equal to the negative eigenvalues of the operator $-\frac{d^{2}}{d x^{2}}+q(x)$ in $L^{2}(0,1)$ with the homogeneous boundary conditions (3.43), that is, boundary conditions $v_{x}(0, \lambda)=v_{x}(1, \lambda)=0$. As it was done in the proof of Theorems 3.1 and 3.3, for all $\lambda$ for which problems (3.42) (3.43) with potentials $q_{1}$ and $q_{2}$ are solvable, one derives the orthogonality relation

$$
\int_{0}^{1} p(x) \psi_{1}(x, \lambda) \psi_{2}(x, \lambda) d x=0,
$$

which actually holds for all $\lambda$ because its left-hand side is an entire function of $\lambda$. Property $\mathrm{C}$ (Theorem 2.2 ) and this orthogonality relation imply $p(x)=0$. Theorem 3.7 is proved

Let us sketch an alternative proof, although it is a longer one. From (3.43) and (3.44) it follows that $c(\lambda) \psi_{x}(1, \lambda)=B(\lambda)$ and $c(\lambda) \psi(1, \lambda)=A(\lambda)$. Thus the function $G(\lambda):=\frac{\psi_{x}(1, \lambda)}{\psi(1, \lambda)}$ is known for all $\lambda$, except possibly at a discrete set, provided that $A(\lambda)$ and $B(\lambda)$ are known. These two functions are known since we assume in problem (IP4) that the functions $a(t)$ and $b(t)$ are known. Since the function $G(\lambda)$ is meromorphic, and known everywhere except possibly at a discrete set, it is known everywhere. The zeros of the function $G(\lambda)$ are the eigenvalues of the problem (3.42) with boundary conditions $v_{x}(0, \lambda)=v_{x}(1, \lambda)=0$, while the poles of $G$ are the eigenvalues of the problem (3.42) with boundary conditions $v_{x}(0, \lambda)=v(1, \lambda)=0$. Therefore the function $G(\lambda)$ determines uniquely the two spectra of the operator in (3.42). By the classical result concerning uniqueness of recovery of $q(x)$ from two spectra (with the same boundary condition on one of the ends of the interval) (see, e.g., [3], [4] or [7]) the conclusion of Theorem 3.7 follows. Note that other boundary conditions can be treated similarly.

Theorem 3.7 is proved in [1: pp. $159-164\}$ by a different argument and with additional assumptions on $b(t)$ and $q(x)$. In [1] the uniqueness theorem for inverse problem of finding the potential from the knowledge of two spectra was used. 


\section{Classical inverse problems}

In this section we give a very short proof of the uniqueness of the solutions to two classical inverse problems:

a) inverse scattering on the half-axis, and

b) inverse spectral problem.

We will give a proof for problem a), and the proof for problem b) with $q \in L_{1,1}\left(\mathbb{R}_{+}\right)$ is the same, because, as shown in [7], in both problems the data are equivalent to the following data:

$$
\left\{f(k), \lambda_{j}, s_{j}, 1 \leq j \leq J\right\} .
$$

Here $f(k)$ is the Jost function, $\lambda_{j}$ are positive numbers which determine the bound states of the corresponding one-dimensional Schroedinger operator on the half-axis, and $s_{j}$ are the normalizing constants defined in [7: p. 255/Formula (53)]. We wish to prove the following

Theorem 4.1. If $q_{m}(m=1,2)$ generate the same data $(4.1)$, then $q_{1}=q_{2}$.

Proof. We assume that $q_{m} \in L_{1,1}\left(\mathbb{R}_{+}\right)$. Recall that $\lambda_{j}$ and the number $J$ in (4.1) are uniquely defined by $f(k)$, which is an analytic in $\mathbb{C}_{+}$function. Namely, $i \lambda_{j}$ are the zeros of $f(k)$ and $J$ is the number of these zeros. Therefore the data (4.1) can be written as

$$
\left\{f(k), s_{j}, 1 \leq j \leq J\right\} .
$$

Denote by $f_{m}(r, k)$ the Jost solution (see formula (2.5)) corresponding to the potential $q_{m}(r)$. Then (3.8) holds and we wish to derive (3.11) (with $x=r$ ). To do this, we derive (3.13) and then use Theorem 2.1 to obtain (3.11). The basic point is to prove that the non-integral term in (3.12) vanishes if the data (4.2) are the same for $q_{1}$ and $q_{2}$. This term can be written as

$$
-f_{2}^{\prime}(0, k) f_{1}(k)+f_{2}(k) f_{1}^{\prime}(0, k)
$$

since at infinity the boundary term in (3.12) vanishes. If the data (4.2) are the same for $q_{1}$ and $q_{2}$, then $f_{1}(k)=f_{2}(k)$, and what remains to be proved is that

$$
f_{1}^{\prime}(0, k)=f_{2}^{\prime}(0, k)
$$

Indeed, if (4.4) is proved, then quantity (4.3) vanishes, and the proof is complete.

However, (4.4) is a direct consequence of the assumption that the data (4.1) for $q_{1}$ and $q_{2}$ are the same. We shall now prove this last claim and then the proof is finished.

To prove that $f_{1}^{\prime}(0, k)=f_{2}^{\prime}(0, k)$, start with the Wronskian formula $f^{\prime}(0, k) f(-k)-$ $f(k) f^{\prime}(0,-k)=2 i k$ and write it as $f^{\prime}(0, k)=S(-k) f^{\prime}(0,-k)+\frac{2 i k}{f(-k)}$. This Riemann problem is solvable by construction. If we prove that it has at most one solution, then the proof of Theorem 4.1 is complete. Suppose there are two solutions $w_{1}$ and $w_{2}$, 
$w_{m}:=f_{m}^{\prime}(0, k)(m=1,2)$, note that, as was proved above, $f_{1}(k)=\cdot f_{2}(k):=f(k)$; and let $w:=w_{1}-w_{2}$. Then

$$
\frac{w(k)}{f(k)}=\frac{w(-k)}{f(-k)}, \quad w(\infty)=0
$$

The relation $w(\infty)=0$ can be easily derived from formula (2.5) and from the relation $f_{1}(k)=f_{2}(k)$, which we have already proved. Moreover, $\frac{w(k)}{f(k)}$ is analytic in $\mathbb{C}_{+}$because $f_{1}^{\prime}\left(0, i k_{j}\right)=f_{2}^{\prime}\left(0, i k_{j}\right)$, as follows from the assumption that $s_{j}$ are the same for $f_{1}$ and $f_{2}$. Recall that [7: p. 255/Formula (53)]

$$
s_{j}=\frac{-2 i k_{j}}{\dot{f}\left(i k_{j}\right) f^{\prime}\left(0, i k_{j}\right)}, \quad \dot{f}:=\frac{\partial f}{\partial k} .
$$

so that $f^{\prime}\left(0, i k_{j}\right)$ are uniquely determined by $s_{j}$ and $f(k)$. Therefore the Riemann problem $\left(4.4^{\prime}\right)$ implies $\frac{w(k)}{f(k)}=0$, and $w(k)=0$. Theorem 4.1 is proved

An alternative proof of the relation $f_{1}^{\prime}(0, k)=f_{2}^{\prime}(0, k)$ can be based on the Marchenko cquation as follows. Note that if the data (4.1) are the same, then the function

$$
F(r):=\sum_{j=1}^{J} s_{j} e^{-\lambda_{j} r}+\frac{1}{2 \pi} \int_{-\infty}^{\infty}\left[1-\frac{f(-k)}{f(k)}\right] e^{i k r} d k
$$

is the same for $q_{1}$ and $q_{2}$. One has (see [7: p. 254/Formula (49)])

$$
f^{\prime}(0, k)=i k-A(0,0)+\int_{0}^{\infty} A^{\prime}(y) e^{i k y} d y
$$

where $A^{\prime}(y):=A_{x}(0, y), A(0,0)=-\lim _{k \rightarrow \infty} i k[f(k)-1]$, and $A(x, y)$ is the kernel from formula (2.5), in which the subindex $j$ is dropped (or [7: p. 254/Formula (49)]). Thus, (4.4) is proved if one proves that

$$
B_{1}=B_{2}
$$

where

$$
B_{m}:=\dot{A}_{m}^{\prime}(y) \quad(m=1,2) .
$$

To prove (4.7), differentiate the Marchenko equation

$$
F(x+y)+A(x, \dot{y})+\int_{x}^{\infty} A(x, t) F(t+y) d t=0 \quad(y \geq x \geq 0)
$$

(see [7: p. 267/Equation (23)]), take $x=0$ and get

$$
F^{\prime}(y)+A^{\prime}(y)-A(0,0) F(y)+\int_{0}^{\infty} A^{\prime}(t) F(t+y) d t=0 .
$$

If the data are the same, then $F^{\prime}(y)$ and $A(0,0)$ are the same. Subtract from equation (4.9) corresponding to $q=q_{1}$ this equation corresponding to $q=\dot{q}_{2}$, to get

$$
B(y)+\int_{0}^{\infty} B(t) F(t+y) d t=0 .
$$

Here $B:=B_{1}-\dot{B_{2}}$. Equation (4.10) has only the trivial solution (see, e.g., [7: p. 269]). Thus, $B=0$, and the proof is complete

The argument used in the proof of Theorem 4.1 is borrowed from [12]. 


\section{Inverse problems with mixed data}

Suppose $q \in L^{1}[0,1]$ is real-valued, $u$ solves $(2.1)$ on the interval $[0,1], u(0, k)=0$ (or $\left.u^{\prime}(0, k)=h_{0} u(0, k)\right)$ and $u(1, k)=0$ (or $\left.u^{\prime}(1, k)+h_{1} u(1, k)=0\right)$, where $h_{0}$ and $h_{1}$ are some constants. Let $\left\{\lambda_{n}\right\}_{n \geq 0}$ be the eigenvalues of the operator (2.1) corresponding to the above boundary conditions, which we take, for example, to be the Dirichlet conditions. Fix an arbitrary number $0<b<1$, assume that $q(x)$ is known for $b \leq x \leq 1$ and the set $\mu_{n}:=\left\{\lambda_{m(n)}\right\}_{n \geq 0}$ is known, where $\lim _{n \rightarrow \infty} \frac{m(n)}{n}=\sigma^{-1}, \sigma>0$. We first consider in Theorem 5.1 the simpler case $\sigma>2 b$ and then treat the more difficult limiting case $\sigma=2 b$.

Theorem 5.1. If $\sigma>2 b$, then the above data determine $q(x)$ on $[0, b]$ uniquely.

Proof. Suppose there are two potentials $q_{1}, q_{2}$ with the same data. Then, as in the proof of Theorem 3.1, one gets

$$
\int_{0}^{b} p(x) \varphi_{2}(x, k) \varphi_{2}(x, k) d x:=g(k)=0 \quad \text { at } k=k_{n}:=\lambda_{m(n)}^{1 / 2}, p:=q_{1}-q_{2}
$$

Here $\ell_{j} \varphi_{j}=0, \varphi_{j}(0, k)=0 \quad(j=1,2), \varphi_{j}:=\frac{\phi_{j}}{k}$, where $\phi_{j}$ are defined by formula (2.14). It follows from (2.14) and (5.1) that $g(k)$ is an entire function of exponential type and $|g(k)| \leq c \frac{e^{2 b|1 m k|}}{1+|k|^{2}}$. Denote by $n(r)$ the number of zeros of $g(k)$ in the disk $|k| \leq r$, and by

$$
h(\theta):=h_{g}(\theta):=\varlimsup_{\lim _{r \rightarrow \infty}} \frac{\ln \left|g\left(r e^{i \theta}\right)\right|}{r} \leq 2 b|\sin \theta|
$$

the indicator of the entire function $g(k)$. It is known [2: Formula (4.16)] that

$$
\varliminf_{r \rightarrow \infty} \frac{n(r)}{r} \leq \frac{1}{2 \pi} \int_{0}^{\pi} h(\theta) d \theta \leq \frac{4 b}{\pi}
$$

where the estimate $|h(\theta)| \leq 2 b|\sin \theta|$ was used. Since (for the Dirichlet conditions)

$$
\lambda_{m(n)}^{1 / 2} \sim \pm m(n) \dot{\pi} \sim \pm \frac{n \pi}{\sigma} \quad \text { as } n \rightarrow \infty,
$$

one gets from (5.2) the inequality $\frac{2 \sigma}{\pi} \leq \frac{4 b}{\pi}$ or

$$
\sigma \leq 2 b
$$

Thus, if $g(k) \not \equiv 0$, then $\sigma \leq 2 b$. Therefore, if $\sigma>2 b$, then $g(k) \equiv 0$, and Theorem 2.2 inplies $p=0$, i.e. $q_{1}=q_{2}$. Theorem 5.1 is proved

Below we consider the case $\sigma=2 b$. Let us discuss some of the applications of Theorem 5.1.

Theorem 5.1 with $\sigma>2 b$ implies rough versions of the results in [14], and the limiting case of Theorem 5.1 with $\sigma=2 b$ yields many of the results in [14]. The limiting case $\sigma=2 b$ is treated in Theorem 5.2 below. Suppose that $q(x)$ is known on $\left[\frac{3}{4}, 1\right]$, that is, $b=\frac{3}{4}$, and $\sigma>\frac{3}{2}$. Then $q(x)$ is uniquely determined on $\left[0, \frac{1}{4}\right]$. In particular, Theorem 
5.2, proved below, yields the classical Borg's theorem about uniqueness of the recovery of the potential and of the boundary conditions for the one-dimensional problem on a finite interval from two spectra, and the result of Hochstadt-Lieberman (see [14]) about the uniqueness of the recovery of the potential if this potential is known on a halfinterval and one spectrum is known. By spectrum here we mean the set of eigenvalues corresponding to some selfadjoint boundary conditions of the type mentioned above. In [14] the main tool is the Weyl function and its analytic properties.

Let us consider now the limiting case $\sigma=2 b$. We will need for this case the additional assumption

$$
m(n)=\frac{n}{\sigma}\left[1+O\left(\frac{1}{n^{\gamma}}\right)\right] \quad(\gamma>1)
$$

Define $G(z):=\prod_{n=1}^{\infty}\left(1-\frac{z}{k_{n}^{2}}\right), z=k^{2}$. Then $w(z):=g(z) G^{-1}(z)$ is an entire function, $|g(z)|<c_{1} \frac{\exp \left(2 b \mid \operatorname{Im} z^{\frac{1}{2}}\right)}{(1+|z|)}$ and the order of $G$ equals $\frac{1}{2},|G(z)|<c_{2} \exp \left(c_{3}|z|^{\frac{1}{2}}\right)$, where $c_{j}>0$ are constants and Im is the imaginary part. The estimate for $G$ is a consequence of the known result that the order of an entire function which is a canonical product constructed from its zeros $z_{n},\left|z_{n}\right| \rightarrow \infty$, equals to the exponent of convergence of the series $\sum_{n=1}^{\infty}\left|z_{n}\right|^{-s}$, where the exponent of convergence is the infimum of $s>0$ for which the above series converges (see [2: Section I.5]). Since $k_{n}^{2} \sim c n^{2}$ as $n \rightarrow \infty$, it follows that the order of $G$ equals $\frac{1}{2}$, as claimed above.

A Phragmen-Lindelö theorem (see [2: Section I.14]) says:

If an entire function $|w(z)| \leq c e^{c_{2}|z|^{\rho}} \quad(0<\rho<1)$ and $\sup _{y \in \mathbb{R}}|w(i y)| \leq c$, then $w(z)=$ const, and if, in addition, $w(i y) \rightarrow 0$ as $y \rightarrow \infty$, then $w(z) \equiv 0$.

The functions $g, G$ are of order $\rho=\frac{1}{2}<1$ and therefore $u$ is of order $\rho \leq \frac{1}{2}$ (see [2: Section I.9]). Moreover, one can derive the estimate

$$
|w(i y)|=\left|g(i y) G^{-1}(i y)\right| \leq \frac{c}{|y|^{\frac{1}{2}}} \quad \text { as } \quad|y| \rightarrow \infty
$$

Thus $w(z) \equiv 0$ by the above Phragmen-Lindelöf theorem. Therefore $g(z)=0$, and, by property $\mathrm{C}$ (Theorem 2.2), $p(x)=0$. We have established the conclusion of Theorem 5.1 also in the limiting case, $\sigma=2 b$ under the additional assumption (5.5).

Let us outline a proof of estimate (5.6). This estimate is a consequence of the following lemma.

Lemma 5.1. If condition (5.5) holds and $\sigma=2 b$, then the estimate

$$
T:=\left|\frac{\sin \left(\sigma(i y)^{\frac{1}{2}}\right)}{\sigma(i y)^{\frac{1}{2}}} G^{-1}(i y)\right|<c
$$

is valid.

Here and below $c>0$ stand for various constants. 
Proof of Lemma 5.1. To check (5.7), denote $\nu_{n}:=\frac{n^{2} \pi^{2}}{\sigma^{2}}$ and use the infinite products for the function $\frac{\sin \left(\sigma(i y)^{\frac{1}{2}}\right)}{\sigma(i y)^{\frac{1}{2}}}$ and for the function $G$. This yields

$$
T^{2}=\prod_{n=1}^{\infty} \frac{1+\frac{y^{2}}{\nu_{n}^{2}}}{1+\frac{y^{2}}{\mu_{n}^{2}}}
$$

If $a>c>0$, then $\frac{1+a}{1+c}<\frac{a}{c}$, and if $0<a \leq c$, then $\frac{1+a}{1+c} \leq 1$. Therefore

$$
T^{2} \leq \prod_{n=1, \frac{\mu_{n}^{2}}{\nu_{n}^{2}} \geq 1}^{\infty} \frac{\mu_{n}^{2}}{\nu_{n}^{2}}<\infty
$$

provided that

$$
\frac{\mu_{n}^{2}}{\nu_{n}^{2}}=1+a_{n} \quad \text { where } \quad \sum_{n=1}^{\infty}\left|a_{n}\right|<\infty .
$$

The Dirichlet eigenvalues $\lambda_{n}$ of the Schrödinger operator on the interval $[0,1]$ have asymptotics

$$
\lambda_{n} \sim(n \pi)^{2}+c+O\left(\frac{1}{n}\right)
$$

where $c$ is some constant. Thus

$$
\mu_{n} \sim \frac{(n \pi)^{2}\left[1+O\left(\frac{1}{n^{\top}}\right)\right]}{\sigma^{2}}+c+O\left(\frac{1}{n}\right) \quad(\gamma>1)
$$

so that (5.8) holds if $\gamma>1$, which is assumption (5.5). Therefore (5.7) is established and the proof of Lemma 5.1 is complete

An estimate similar to (5.7) is used in [14].

Let us formulate the result we have proved:

Theorem 5.2. If $\sigma \geq 2 b$ and (5.5) holds, then the knowledge of $q(x)$ on the interval $[b, 1]$ and of the subset of eigenvalues $\left\{\lambda_{m(n)}\right\}_{n \geq 0}$ determine $q(x)$ on $[0, b]$ uniquely.

An alternative proof of Theorem 5.2 can be sketched: there exists a sequence of circles $|z|=r_{n} \rightarrow \infty$ such that $w(z) \rightarrow 0$ as $|z|=r_{n} \rightarrow \infty$ provided (5.5) holds; existence of such a sequence is discussed in [4: pp. 33, 242], Lemma 3.4.2 in [4: p. 242) can be used in this context. From this and the maximum principle it follows that $w(z) \equiv 0$. The rest of the argument is the same as above. 


\section{References}

[1] Denisov, A. M.: Introduction to the Theory of Inverse Problems (in Russian). Moscow: Univ. Moscow 1994.

[2] Levin, B.: Distribution of Zeros of Entire Function. Providence (RI): Amer. Math. Soc. 1980.

[3] Levitan, B. M.: On the completeness of the products of solutions of two Sturm-Liouville equations. Diff. Int. Eq. 7 (1994), 1 - 14.

[4] Marchenko, V. A.: Sturm-Liouville Operators and Applications. Boston: Birkhauser 1986.

[5] Ramm, A. G.: On completeness of the products of harmonic functions. Proc. Amer. Math. Soc. 99 (1986), 253 - 256.

[6] Ramm, A. G.: Completeness of the products of solutions to PDE and uniqueness theorems in inverse scattering. Inverse Problems 3 (1987), L77 - L82.

[7] Ramm, A. G.: Multidimensional Inverse Scattering Problems. New York: Longman/Wiley 1992. Russian translation of the expanded monograph by Mir Publishers (Moscow) 1994

[8] Ramm, A. G.: Recovery of the potential from I-function. Math. Reports Acad. of Sci. Canada 9 (1987), 177 - 182.

[9] Ramm, A. G.: Inversion of fixed-frequency surface data for layered medium. J. Inv. and Ill-Posed Problems 2 (1994), 263 - 268.

[10] Ramm, A. G.: Recovery of compactly supported spherically symmetric potentials from the phase shift of s-wave. In: Spectral and Scattering Theory (ed.: A. G. Ramm). New York: Plenum Publ. 1998, pp.111 - 130.

[11] Ramm, A. G.: Compactly supported spherically symmetric potentials are uniquely determined by the phase shift of s-wave. Phys. Lett A 242 (1998)4-5, $215-219$.

[12] Ramm, A. G.: A new approach to the inverse scattering and spectral problems for the Sturm-Liouville equation. Ann. Phys. 7 (1998), 321 - 338.

[13] Ramm, A. G. and G. Porru: Completeness and non-completeness results for the set of products of solutions to differential equations. Appl. Anal. 60 (1996), $241-249$.

[14] Rio, R., Gesztesy, F. and B. Simon: Inverse spectral analysis with partial information on the potential. IMRN 15 (1997), $751-758$.

Received 12. 07. 1998; in revised form 02.03.1999 